\title{
Improvement of Detection in Computed Radiography by New Single-Exposure Dual-Energy Subtraction
}

\author{
Wataru Ito, Kazuo Shimura, Nobuyoshi Nakajima, Masamitsu Ishida, and Hisatoyo Kato
}

\begin{abstract}
It is reported that the use of the dual-energy subtraction method enhances the abnormal shadow detection capability. However, because the subtracted image is significantly inferior to the original in signal-to-noise ratio (SNR), the $x$-ray dosage normally used for chest $x$-rays has not yielded subtracted images with adequate SNRs. Under these circumstances, we focus on the fact that there is a correlation between the noise contents of bone-and soft-tissue-subtracted images although there is no correlation between the signal contents of these images. We propose an algorithm that improves SNRs of subtraction images by reducing the noise only.
\end{abstract}

KEYWORDS: computed radiography, photostimulable phosphor, image processing.

$\mathbf{M}$ ANY RESEARCH institutes are conducting research on computer-aided image processing with a view toward image diagnostic value enhancement because the computed radiography using photostimulable-phosphor imaging plate (IP) is developed ${ }^{1}$ to permit digital image generation that excels in linearity and reproducibility. Under such circumstances, the dual-energy subtraction method ${ }^{2}$ is attracting a good deal of attention. It is expected to improve the diagnostic detection capability as it eliminates the shadows produced by particular substances and therefore allow the physician to describe abnormalities overlapping with bone shadows. ${ }^{3}$

The dual-energy subtraction method is roughly divided into the dual-exposure technique ${ }^{4}$ and single-exposure technique. ${ }^{5,6}$ The dual-exposure technique is used to generate two different images by making two exposures at different

From the Technology Development Center, Miyanodai, Fuji Photo Film, Japan.

Address reprint requests to Wataru Ito, Miyanodai Technology Development Center, Fuji Photo Film Company, Ltd, 798, Miyanodai, Kaisei-Machi, Ashigarakami-Gun, Kanagawa, 258 Japan.

Reprinted with permission from Medical Imaging VI: Picture Archiving and Communications Systems, Society of PhotoOptical Instnimentation Engineers, 1992.

Copyright 11992 by The Society of Photo-Optical Instrumentation Engineers

0-8194-0804-2/92/\$4.00 tube voltage settings. The single-exposure technique, however, is used to generate two different images by making only one exposure while a filter attenuating low-energy component is sandwiched between two detectors. When the singleexposure technique is used, however, the noise of the back image increases because the filter that is used provides considerable $x$-ray attenuation. ${ }^{7}$ As the subtraction process enhances the subtle contrast difference between high- and low-energy images, the noise of subtracted images is seriously increased. Although the subtraction process is said to improve the diagnostic detection capability, ${ }^{8}$ it is still not widely used for clinical applications because adequate subtracted image quality is not obtained at $\mathrm{x}$-ray dosage settings that are normally used for chest examination. When the dual-exposure technique is used, however, artifacts may appear that are caused by movement because the two images are derived from differently timed exposures. With the dual-exposure technique it is therefore difficult to obtain subtracted images that depict blood vessel shadows and other minute structures. ${ }^{9}$

We have developed a new subtraction algorithm that provides improved signal-to-noise radio (SNR) on the basis of two different images derived from the conventional singleexposure dual-energy subtraction technique.

\section{MATERIAL AND METHODS}

\section{Imaging System}

$\mathrm{A} \mathrm{Cu}$ filter of $0.8-\mathrm{mm}$ thickness is sandwiched between two IPs. With this $\mathrm{Cu}$ filter and IP combination placed in a cassette, the IPs are exposed to $x$-rays at $100 \mathrm{kV}$ (Fig 1). The front IP then records the $x$-ray information transmitted through the object, whereas the back IP records the $\mathrm{x}$-ray information whose low-energy component is attenuated by the $\mathrm{Cu}$ filter. Therefore, one $\mathrm{X}$-ray exposure causes the front IP to record a low-energy image and the back IP to record a high-energy image.

The information recorded in the IPs is read by the Fuji Computed Radiography system (FCR7000, Fuji Photo Film, Tokyo, Japan) and stored on optical disk as digital images. The two images saved on optical disk are transferred to the Sun SPARC station IPX (Sun Microsystems, Mountain View, CA) and processed. The processed images are saved again onto optical disk and then recorded onto film by the 


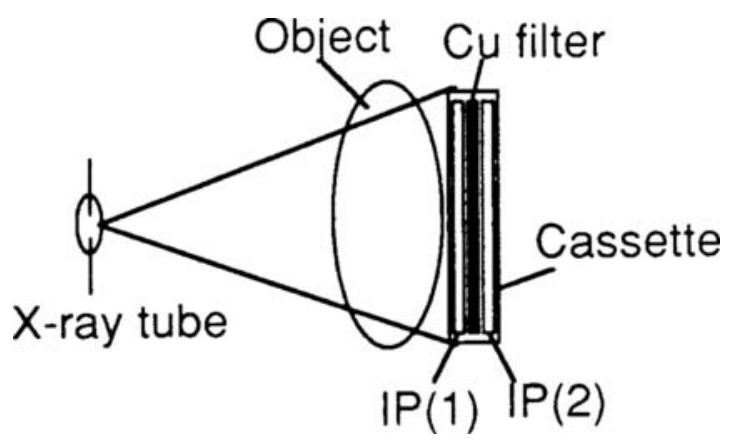

Fig 1. Schematic layout of the single exposure dual-energy imaging technique.

Image Printer (Fig 2). In this study, images having a pixel size of $0.2 \times 0.2 \mathrm{~mm}(1,760 \times 1,760$ pixels $)$ and a density resolution of 10 bits are processed using $14 \times 14$-inch IPs.

\section{Algorithm}

The new algorithm is formulated to achieve the following three aims: (1) to obtain noise-reduced subtracted images by accurately registering the low-and high-energy images; (2) to perform weighted subtraction to entirely eliminate the signal content resulting from bone or soft tissue; and (3) to reduce only the noise without deteriorating the bone or soft-tissue signai. To accomplish the third target, ie, noise reduction, a repetitive processing algorithm is used to alternately obtain bone-and soft-tissue-subtracted images (Fig 3). The details of the new subtraction process are given below.

Registration. Two images are registered by detecting the position coordinates of two doughnut-shaped metal markers impressed at the time of exposure. The low-energy image derived from the front IP and the high-energy image derived from the back IP differ in distance to the object. Therefore, the high-energy image is slightly magnified in comparison with the low-energy one. To cope with this situation, the high-energy image is subjected to affine transformation with the coefficient calculated from the marker position coordinates so that the two images are registered by image rotation, shifting, and reduction.

Weighted subtraction performed in consideration of beam hardening. Dual-energy subtraction makes use of the fact that the low- and high-energy images differ in contrast because of their energy distribution difference. Therefore, the weighting coefficient is determined to eliminate bone or

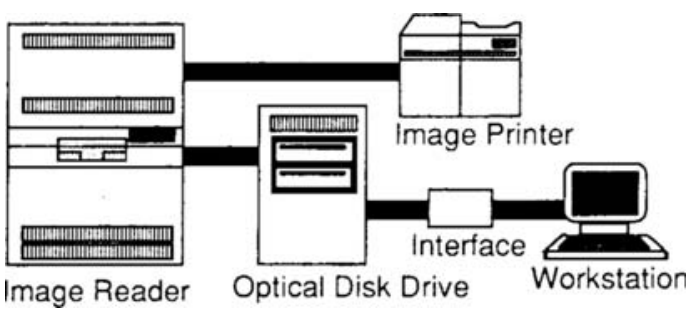

Fig 2. System components for image processing.

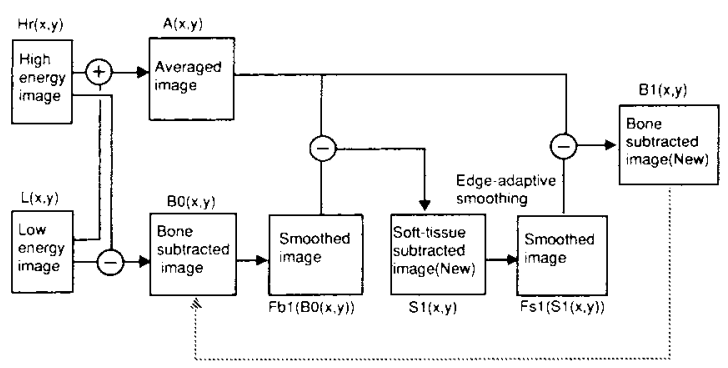

Fig 3. Principle of the new dual-energy subtraction process.

soft-tissue shadows. In actual radiography, beam hardening occurs when $\mathrm{x}$-rays are transmitted through the object, and the degree of beam hardening depends on the object's thickness. For example, x-ray transmissions through the lung field and mediastinum result in differing energy distributions. For bone or soft-tissue elimination, therefore, it is necessary to use different weighting coefficients for the lung field and mediastinum.

To determine the degree of beam hardening, a smoothed image is derived from the low-energy image using a mask of $127 \times 127$ pixels $(25.4 \times 25.4 \mathrm{~mm})$. In this smoothed image, the lung field density is high whereas the mediastinum density is low. The densities of the smoothed image are correlative to the degree of beam hardening. Consequently, the influence of beam hardening can be compensated for by determining the dual-energy subtraction weighting coefficient in accordance with the smoothed image density. More specifically, the following calculation is performed:

$$
\begin{aligned}
& \mathrm{B} 0(\mathrm{x}, \mathrm{y})=\mathrm{ka}[\operatorname{Lu}(\mathrm{x}, \mathrm{y})] \times \operatorname{Hr}(\mathrm{x}, \mathrm{y}) \\
& \quad-\mathrm{kb}[\mathrm{Lu}(\mathrm{x}, \mathrm{y})] \times \mathrm{L}(\mathrm{x}, \mathrm{y})+\mathrm{kc}[\operatorname{Lu}(\mathrm{x}, \mathrm{y})], \quad \text { (Equation 1) }
\end{aligned}
$$

where $B O$ is the bone subtracted image; $H r$ is the registered high-energy image; $L$ is the low-energy image; $L u$ is the smoothed low-energy image; and $k a ; k b$; and $k c$ are parameter tables for subtraction.

Weighted averaging for highest degree of noise reduction. For the process of subtracted image noise reduction, the original image, which serves as the reference, is needed. The low-energy image can be used as the original image. However, because the noise of the original image becomes the lower limit for reducing the noise of the subtracted image, the original image that was used should be a weighted-average image that represents the highest degree of noise reduction. When the low-energy imge noise is $N L$ and the high-energy image noise is $N H$, the following calculation should be performed to minimize the noise of the weighted average image $A$ :

$$
\begin{aligned}
A(x, y)= & \frac{(N L)^{2}}{(N H)^{2}+(N L)^{2}} \times \operatorname{Hr}(x, y) \\
& +\frac{(N H)^{2}}{(N H)^{2}+(N L)^{2}} \times L(x, y)
\end{aligned}
$$

(Equation 2)

Deriving a soft-tissue-subtracted image from a bone-subtracted image. The weighted-average image consists of a bone signal and a soft-tissue signal. The bone-subtracted 
image consists of a soft-tissue signal only. Therefore, the following equation is obtained:

$$
S 1(x, y)=A(x, y)-B 0(x, y),
$$

where $S l$ is the soft-tissue subtracted image.

However, the SNR of bone-subtracted images obtained with the conventional method is decreased because the noise is more enhanced when compared with the signal in the process of subtraction. Therefore, noise is superposed on the soft-tissue-subtracted image that is determined above. When this matter is examined from a different viewpoint, it is found that there is a close correlation between bone- and soft-tissue-subtracted image noises. ${ }^{10.11}$ That is, the SNR of soft-tissue-subtraced image can be improved by reducing the noise of bone-subtracted image with a smoothing filter.

$$
\mathrm{S} 1(\mathrm{x}, \mathrm{y})=\mathrm{A}(\mathrm{x}, \mathrm{y})-\mathrm{Fb} 1[\mathrm{~B} 0(\mathrm{x}, \mathrm{y})], \quad \text { (Equation 4) }
$$

where $F b 1$ is the smoothing filter.

Because as the high-frequency component of the softtissue signal in the bone-subtracted image $(B O)$ is small, the high-frequency component in $\mathrm{B} 0$ is mostly noise. Therfore, a low-pass filter $(F b l)$, which averages the results of several-pixel masking, is brought into action for noise reduction purposes. The noise in the filtered image $[\mathrm{Fb} 1(\mathrm{~B} 0)]$ and the soft-tissue-subtracted image $(S 1)$ is reduced by $\mathrm{Fb} 1$. However, the bone signal in $\mathrm{S} 1$ is not deteriorated because Fb1 reduced only the high-frequency components of the noise and the soft-tissue signal in B0.

Deriving a bone subtracted image from a soft-tissue-subtracted image. In nearly the same manner as indicated in the previous section, it is possible to derive a bonesubtracted image from a weighted-average image and a soft-tissue-subtracted image.

$$
B 1(x, y)=A(x, y)-\operatorname{Fs} 1[S 1(x, y)],
$$

(Equation 5)

where $F_{S} l$ is the smoothing filter.

The difference is that a simple low-pass filter cannot be used as the smoothing filter. The smoothing filter separates noise from the bone signal of the soft-tissue-subtracted image and decrease the noise only. The bone signal has the high-frequency component for bone edges and the lowfrequency component for nearly all the remaining area. However, the noise is completely random. With our attention focused on these characteristics, we use an edgeadaptive smoothing filter ( $F s 1$ ) that cuts of the middle and high-frequency components without causing edge deterioration. This process reduces the middle and high-frequency component of the noise and does not deteriorate the soft-tissue signal in the bone-subtracted image (B1).

Noise and artifact reduction by repetitive processing. As stated earlier, the noise of bone-and soft-tissue-subtracted images can be reduced by using soft-tissue- and bonesubtracted images, respectively. However, because no smoothing filters can reduce noise only without deteriorating the bone or soft-tissue signal, the bone signal is superposed on the bone-subtracted image as an artifact and the soft-tissue signal is superposed on the soft-tissuesubtracted image as an artifact, therefore, the following repetitive processing operations are performed to accomplish both noise and artifact reduction (Fig 3 ).
(Step 1)

$$
\mathrm{S} 1(\mathrm{x}, \mathrm{y})=\mathrm{A}(\mathrm{x}, \mathrm{y})-\mathrm{Fb} 1[\mathrm{~B} 0(\mathrm{x}, \mathrm{y})] . \quad \text { (Equation 6) }
$$

In $S 1$, the high-frequency component of noise is reduced by $\mathrm{Fb} 1$. However, the high-frequency component of the soft-tissue signal is partly superposed on $\mathrm{S} 1$ as an artifact.

(Step 2)

$$
\mathrm{B} 1(\mathrm{x}, \mathrm{y})=\mathrm{A}(\mathrm{x}, \mathrm{y})-\mathrm{Fs} 1[\mathrm{~S} 1(\mathrm{x}, \mathrm{y})] . \quad \text { (Equation 7) }
$$

Fs1 reduces the high-frequency component of the softtissue signal superposed on $\mathrm{S} 1$ as an artifact and the middleand high-frequency components of noise. Because the noise is still high at this stage, the edge-adpative filter does not properly detect the edge so that the high-frequency component of the bone signal is partly superposed on B1 as an artifact.

(Step 3)

$$
\mathrm{S} 2(\mathrm{x}, \mathrm{y})=\mathrm{A}(\mathrm{x}, \mathrm{y})-\mathrm{Fb} 2[\mathrm{~B} 1(\mathrm{x}, \mathrm{y})] . \quad \text { (Equation 8) }
$$

$\mathrm{Fb} 2$ reduces not only the high-frequency component of noise but also the high-frequency component of the bone signal superposed on B1 as an artifact. Meanwhile, the high-frequency component of the soft-tissue signal is superposed again on S2. However, as the cut-off frequency of the low-pass filter setting is higher than that in Step 1, the artifact is reduced when compared with $\mathrm{S} 1$.

(Step 4)

$$
B 2(x, y)=A(x, y)-F s 2[S 2(x, y)] . \quad \text { (Equation 9) }
$$

Fs2 reduces not only the middle- and high-frequency components of noise but also the high-frequency component of the soft-tissue signal superposed on S2 as an artifact. Because the noise of $S 2$ is lower than that of $S 1$ in Step 2 and the cut-off frequency of Fs2 is higher than that in Step 2, the bone signal artifact superposed on $\mathrm{B} 2$ is reduced when compared with B1.

$$
\begin{aligned}
& (\operatorname{Step} 2 n-1) \\
& \operatorname{Sn}(x, y)=A(x, y)-\operatorname{Fbn}\left[B_{n-1}(x, y)\right] \quad \text { (Equation 10) }
\end{aligned}
$$
and

(Step 2n)

$$
\operatorname{Bn}(x, y)=A(x, y)-\operatorname{Fsn}[\operatorname{Sn}(x, y)] .
$$

(Equation 11)

When processing is repeated with the filter parameter varied gradually, it is possible to accomplish reducing both noise and artifact in subtracted image.

\section{RESULTS}

\section{Noise Analysis Using the Phantom}

We measured the noise power spectra of a conventional bone-subtracted image and a new bone-subtracted image. Dual-energy images were acquired using the phantom (Fig 4A). Exposure was performed under the condition $(100 \mathrm{kV}, 100 \mathrm{~mA}, 64 \mathrm{mS}-180 \mathrm{~cm}$ focus film 


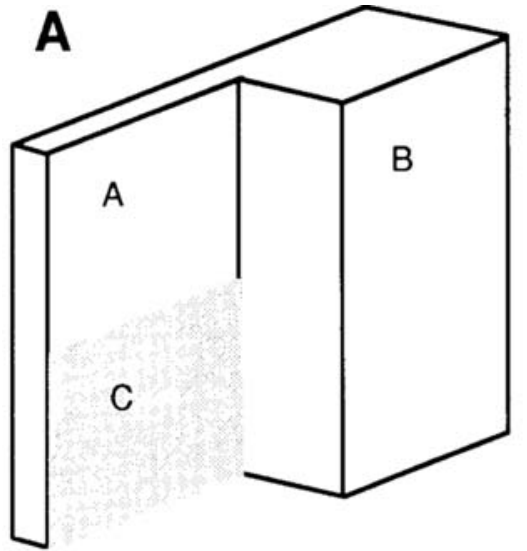

Fig 4. Noise analysis using the phantom: (A) the phantom for noise analysis; $A, 5-\mathrm{cm}$ lucite block that simulates the lung region; $B, 15-\mathrm{cm}$ lucite block that simulates the mediastinum region; and $C, 5-\mathrm{cm}$ lucite block with $2 \mathrm{~mm}$ aluminum plate that simulates the rib region in the lung field. (B) noise power spectra.

distance, 40 lines/cm, 10:1 Bucky grid) that is usually used for chest examination with screen/ film combination of HR-4[Fuji]/HR-S[Fuji]. Noise power spectra of both the conventional and new bone-subtracted images are shown in Fig 4B. Noise power spectra of the proposed bone-subtracted image were greatly improved in the middle- and high-frequency region compared with the conventional bone-subtracted image.

\section{Results of the Clinical Study}

The results of clinical applications are presented in Fig 5. In the low-energy image (Fig $5 \mathrm{~A}$ ), nodules overlapping with ribs are barely visible. In the conventional bone-subtracted image (Fig 5B), a nodule is more easily recognized because rib shadows are eliminated. However, edges are not clear because SNR is not good enough. In the new bone-subtracted image (Fig 5C), however, the nodule edges are clear and lung field blood vessel shadows can be traced to peripheries. When compared with the conventional soft-tissue-subtracted image (Fig 5D), the new soft-tissue-subtracted image (Fig $5 E$ ) clearly depicts the vertebra of mediastinum.
It is evident that the detection capability is enhanced.

\section{CONCLUSION}

Using the new single-exposure dual-energy subtraction algorithm, subtracted images with adequate SNRs were obtained at an x-ray dosage setting normally used for chest examinations. This algorithm requires high accuracy. We have successfully worked out this algorithm because adequate linearity, spatial resolution, density resolution, and reproducibility are provided by the IP and Fuji computed radiography system (FCR). It can therefore be said that the combination of the FCR and new singleexposure dual-energy subtraction algorithm will provide new diagnostic information that is not furnished by the conventional screen/film system. We hope that the new dual-energy subtraction algorithm will find wide applications in medical examinations and contribute to the early detection of diseases. ${ }^{12}$

\section{ACKNOWLEDGMENT}

The authors thank Drs Kozuka, Tamura, and Kido in the Department of Radiology at the Osaka University Medical School for their medical advice and suggestions. 

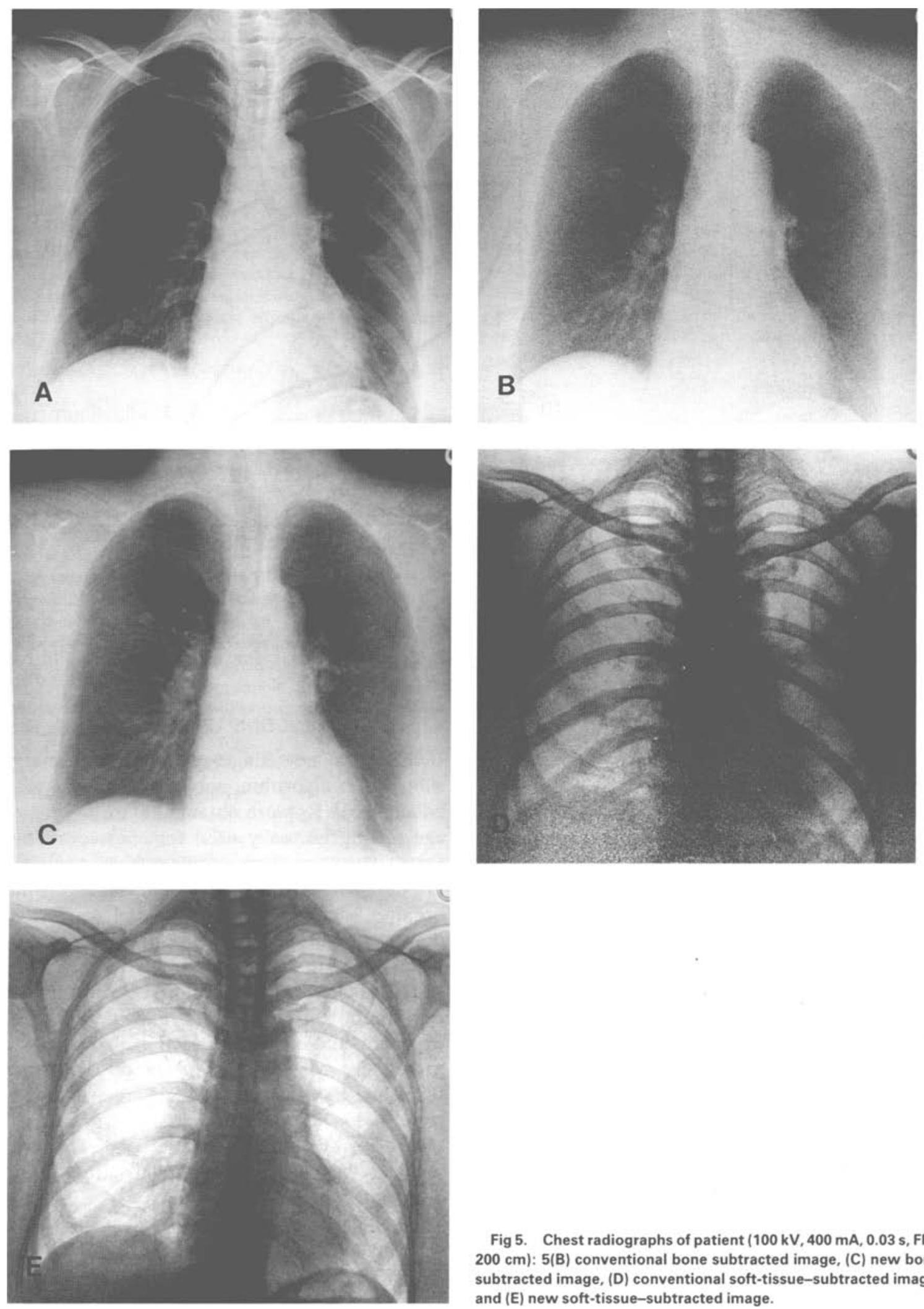

Fig 5. Chest radiographs of patient ( $100 \mathrm{kV}, 400 \mathrm{~mA}, 0.03 \mathrm{~s}$, FFD $200 \mathrm{~cm}$ ): 5(B) conventional bone subtracted image, (C) new bone subtracted image, (D) conventional soft-tissue-subtracted image, and $(E)$ new soft-tissue-subtracted image. 


\section{REFERENCES}

1. Sonoda M, Takano M, Miyahara J, et al: Computed radiography utilizing scanning laser stimulated luminescence. Radiology 148:833-838, 1983

2. Kruger RA, Armstrong JD, Sorenson JA, et al: Dual energy subtraction technique for detecting calcifications in solitary pulmonary nodules. Radiology 140:213-219, 1981

3. Ishigaki T, Sakuma S, Ikeda M: One-shot dual-energy subtraction chest imaging with computed radiography: Clinical evaluation of film images. Radiology 168:67-72, 1988

4. Nisitani H, Umezu Y, Ogawa K, et al: Dual-energy projection radiology using condenser $X$-ray generator and digital radiography apparatus. Radiology 161:533-535, 1986

5. Ishigaki T, Sakuma S, Horikawa Y, et al: One-shot dual-energy subtraction imaging. Radiology 161:271-273, 1986

6. Stewart BK, Huang HK: Single-exposure dual-energy computed radiography. Med Phys 17:866-875, 1990
7. Ho JT, Kruger RA, Sorenson JA: Comparison of dual and single exposure techniques in dual-energy chest radiography. Med Phys 16:202-208, 1989

8. Takashima T: Single exposure energy subtraction chest radiography in the diagnosis of pulmonary cancer. Acta Radiol (Japan) 47:455-464, 1987

9. Takeuchi $H$, Chuang KS, Huang HK: Dual energy imaging in projection radiography. SPIE 626:39-48, 1986

10. Prokop M, Dohring W: A noise reduction method for dual energy CT. Radiology 157(P):339, 1985 (abstr)

11. Kalender W, Klotz E, Kostaridou L: An algorithm for noise suppression in dual-energy CT material density images. IEEE Trans Med Imaging 7:218-224, 1988

12. Kido S, Tamura S, Kozuka T, et al: Improvement of detection with new single-exposure dual-energy images at chest radiography. RSNA '91 Scientific exhibits \#920, 1991 